\title{
Street Network Studies: from Networks to Models and their Representations
}

\author{
Stephen Marshall ${ }^{1}$ id . Jorge Gil $^{2} \cdot$ Karl Kropf $^{3} \cdot$ Martin Tomko $^{4} \cdot$ Lucas Figueiredo $^{5}$
}

Published online: 7 November 2018

(C) The Author(s) 2018

\begin{abstract}
Over the last fifty years, research into street networks has gained prominence with a rapidly growing number of studies across disparate disciplines. These studies investigate a wide range of phenomena using a wealth of data and diverse analytical techniques. Starting within the fields of transport or infrastructure engineering, street networks have commonly been treated as sets of more or less homogeneous linear elements, connecting locations and intersecting at junctions. This view is commonly represented as a graph, which provides a common and rigorous formalisation accessible across disciplines and is particularly well-suited for problems such as flow optimisation and routing. Street networks are, however, complex objects of investigation and the way we model and then represent them as graphs has fundamental effects on the outcomes of a study. Many approaches to modelling street networks have been proposed, each lending itself to different analyses and supporting insights into diverse aspects of the urban system. Yet, this plurality and the relation between different models remains relatively obscure and unexplored. The motivations for adopting a given model of the network are also not always clear and often seem to follow disciplinary traditions. This paper provides an overview of key street network models and the prima facie merits of pertinent alternative approaches. It suggests greater attention to consistent use of terms and concepts, of graph representations and practical applications, and concludes with suggestions for possible ways forward.
\end{abstract}

Keywords Street networks $\cdot$ Modelling $\cdot$ Graph representation

\section{Introduction}

With the increasing urbanisation and the associated relevance of urban studies exploring the environmental, economic, demographic and social dimensions of cities, street

Stephen Marshall

s.marshall@ucl.ac.uk

Extended author information available on the last page of the article. 
networks have become a central object of global scientific interest over the last fifty years. Because street networks support a wide range of urban processes they attract attention from scholars in many disciplines, including transport and urban planners, architects, geographers, environmental psychologists and, recently, physicists. Street network studies include investigations into network structure, connectivity, centrality, circuity, traversal, hierarchy, typology and evolution (e.g., Courtat et al. 2011; Crucitti et al. 2006; Giacomin and Levinson 2015; Jiang and Okabe 2014; Lagesse et al. 2015; Louf and Barthelemy 2014; Masucci et al. 2014; Stavroulaki et al. 2017; Strano et al. 2012; Xie and Levinson 2007; Yerra and Levinson 2005). This cross-disciplinary interest and the plurality of scholarly approaches and purposes is a welcome sign of scientific relevance. Yet the lack of communication between these approaches raises a problem of methodological and terminological fragmentation and entrenchment. There is then the risk of duplicated, contradictory or incommensurable results and a lack of replicability.

The various quantitative, computational studies of street networks predominantly apply methods based on graph theory and network science (Newman 2003; Brandes et al. 2013; Ducruet and Beauguitte 2014; Kivelä et al. 2014). However, as Butts (2009: 416) has pointed out, "To represent an empirical phenomenon as a network is a theoretical act (...) the appropriate choice of representation is key to getting the correct result". Graphs are, in other words, only a mathematical abstraction, a formal representation of a model removed from the physical reality of street environments through a process of abstracting and modelling. This involves generating a simplified representation of the street network by singling out the main elements of study and identifying their relations. Crucially, it determines what will be represented as nodes (vertices) and links (edges) in a graph and what additional parameters of the street network the graph should capture. We call this step network modelling, which is normally embedded in a larger model of a specific phenomenon.

The plurality of approaches is not always evident in the literature. For instance, from Network Analysis in Geography (Haggett and Chorley 1969) to a recent review on spatial networks (Barthelemy 2011), the dominant network model is one that represents the street junctions as vertices in the graph and the linear street segments as its edges. In street network studies, this step is often not commented upon, not necessarily performed as a conscious modelling decision. Yet, it is a selective decision that determines analytical possibilities (Anez et al. 1996; Winter 2002; Meeteren et al. 2016). Thus, in the network modelling step researchers often simply follow disciplinary precedents, unaware of, or unclear about, the diversity of approaches available in other fields of urban research. As a result, they may reproduce past studies inconsistently and under different nomenclature.

Some authors acknowledge alternative street network models, for example (Anez et al. 1996; Batty 2004a; Winter 2002; Porta et al. 2006a, b). These different models are, however, often presented from a narrow, application-specific perspective. Here, we call for a broader reflection on the relative suitability of broad families of network models.

We open up this debate by analysing the different approaches to street network modelling (Section 2), including the consideration of their graph representations and 
the differences in nomenclature and the roles of topology, geometry, directionality and weights (Section 3); and the different types of network data used and the manipulations performed to support diverse analyses (Section 4). In highlighting their purpose and characteristics, we finally argue that widening the range of approaches available to each discipline and clarifying the relations between them opens up opportunities for developing street network studies more comprehensively and effectively (Section 5).

\section{Modelling Street Networks}

\subsection{From Transport Networks to Spatial Networks}

Transport networks are a prominent class of networks studied in the literature (Caldwell 1961; Kansky 1963; Garrison and Marble 1962; Haggett and Chorley 1969; Barthelemy 2011). A given network is generally considered to be an assembly of elements and their relations, a transport network is a spatial network of connected linear elements with either an enduring presence in space, as in the case of road and rail networks, or a network of periodic paths between origins and destinations fixed in space, as in the case of airline and shipping networks. The focus of the models is on linear paths for movement from $A$ to $B$ and the costs associated with this movement (i.e. time, energy, financial). In the most typical conceptualisation as a graph, nodes in a transport network typically represent settlements (such as whole cities, regions or any other kind of origin or destination), individual loci of connection or access (interchanges, junctions, stations) or any other point of transition or termination (including culs-de-sac, network boundary points). Links represent the paths of movement (whether fixed and enduring physical infrastructure or periodic paths through the air or water) between those nodes.

The modelling of transport networks as graphs is a routine and a visually intuitive interpretation when there is a geometric similarity between geographical features and graph elements. The network nodes can be easily represented as graph vertices and the linear links as graph edges. This is, however, not the only possible representation.

\subsection{The Multifaceted Nature of Street Networks}

A road is a physically constructed or demarcated path or channel to accommodate flows of people and vehicles. The connectivity of the road network is assured by junctions, nodes where some event might occur: a change of direction, or conflicts of circulation requiring management to avoid accidents. From this perspective, an urban street network is simply a road network in an urban area: a street can be modelled as a linear element providing a path of movement from one end to the other. In urban studies, however, the role of streets is perceived much more comprehensively.

Viewed broadly, a street is not just a linear conduit, but it may also be a container of urban life, a reflection and expression of civic society, a cultural interface, a political act (Anderson 1978; Gehl 2011; Sadik-Khan 2017). A street is thus a setting 
for human behaviour; the people and activities that exist there, and their social and economic interactions are important characteristics. The physical characteristics of streets are not limited to linear length and width, and movement can exist in a 2D field of the street surface not confined to its medial axis (Hahmann et al. 2018). It can also be influenced by the 3D nature of the street profile or the vistas the street affords. This is particularly true when dealing with pedestrians, for whom a street has a more continuous two- or three-dimensional quality, not lending itself to representation by discrete linear elements; and where there is an intimate relation between the pedestrian space and adjoining buildings (Ståhle et al. 2005; Sevtsuk and Mekonnen 2012), and indeed with the contiguous 'network' of pedestrian routes inside buildings (Mandloi and Thill 2010).

A street - and by extension, the street network - is thus a multi-dimensional object of study in its own right, that cannot be fully understood if only considered as a link in a transport network like any other. Yet, even if transport is the main focus of a study, street systems possess distinctive features that ought to be considered in order to properly understand them as networks.

\subsection{Hierarchical Nature of Street Networks}

A crucial aspect of urban roads and streets is their continuity: a road or street will routinely be continuous through junctions, that is, have a continuous physical presence and identity that affects its function and use by people. We can thus usefully distinguish between main (through) roads and streets that are continuous through junctions; and subsidiary or side streets that are not. The way these streets are connected - some continuous, others terminating - directly gives rise to a particular hierarchical structure typical of road and street networks. This distinction is routinely overlooked in conventional studies that see all links as simply spanning from one node to the next, in which every link is in essence identical and individual links are only distinguished by their relative position in the overall network.

In contrast, if we recognise streets, roads or equivalent paths of movement as continuous entities, they may be distinguished from each other by virtue of being continuous through a greater or lesser number of intermediate junctions. This generates a particular sense of hierarchy that is typically not explicit in street network models where all links simply span between adjacent nodes. Eventually, a sense of hierarchy could be obtained after interpretation of specific kinds of graph analysis. Modelling streets as continuous elements, however, allows for the direct differentiation of types of streets based on configuration (e.g. spine street, connector, loop) (Marshall 2005; Kropf 2008).

If we further consider street-running public transport, continuity also plays a role as stops are part of lines (services), and lines are features of specific modes (bus, tram, metro, or train). In a metro network the location of the individual station (node) has a local impact on the surrounding streets, but the nature of the specific metro line (collection of nodes and links) also has an influence on the attractiveness of a location, and the metro network as a whole exerts a particular influence that is stronger than the presence of only a bus network. These locations have been also 
called transition points and strongly relate to the functional structure of the city as a whole (Tomko and Winter 2013).

\subsection{Alternative Approaches to Modelling Streets}

A street or road can be seen as an aggregation of street segments (hence a composite, or 'second order' element; Courtat et al. 2011; Figueiredo 2015a); it can equally be considered an integral spatial whole that is fragmented into successive street segments or axial lines, or other finer scale elements. From this latter perspective, a street or road is the primary element. The key question then is how to identify and distinguish individual streets or street-like elements.

Several alternative methods have been developed to address this question, with a wide application to urban morphological and movement studies in academia and practice worldwide. The space syntax community pioneered this approach by introducing models based on axial lines (Hillier and Hanson 1984), which can be interpreted as street-like elements (Hillier et al. 1993). Later, models based on line or street segments became increasingly prominent, not least because of a broad availability of data (Turner 2007; Peponis et al. 2008).

In parallel, other methods of modelling the continuity of streets have been developed and applied to empirical studies. Approaches that capture the route structure, using the route as a primary element (Marshall 2005; Kropf 2008); that distinguish streets by name (Jiang and Claramunt 2004a, b); or that create street-like elements based on the angular continuity of either axial lines or street segments: strokes (Thomson and Richardson 1999), continuity lines (Figueiredo and Amorim 2005b), intersection continuity negotiation (ICN) (Porta et al. 2006a), natural roads (Jiang et al. 2008; Tomko et al. 2008). (These techniques are quite similar and always choose the "best continuation" (least angle). However, continuity lines and natural streets also adopt an angular threshold above which street segments or lines are not aggregated.) A further representational approach is that of the line structure - composed of continuous lines and points - mathematically comparable with an incidence structure, and interpretable as having a degree of abstraction between network models and graphs (Marshall 2016). Each approach is a particular interpretation of a street network and has particular purposes, advantages and limitations (Fig. 1).

\section{Graph Representations of Street Networks}

\subsection{From Model to Representation}

In order to apply network analysis algorithms from graph theory on a street network model it must be converted to a graph representation (Fig. 1): a mathematical representation consisting of vertices connected by edges loaded with weights or labels, that can be directed or undirected. The representation of the nodes and links of a transport network (Fig. 1, e) as respectively the vertices and edges in a graph (Fig. 1, f) is the dominant street network graph representation (Anez et al. 1996). This is referred 
(a)

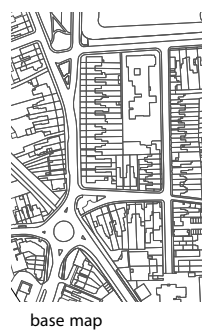

(e)

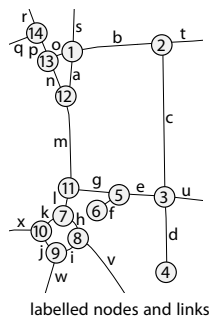

(i)

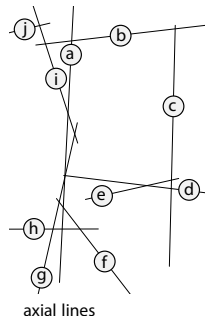

(m)

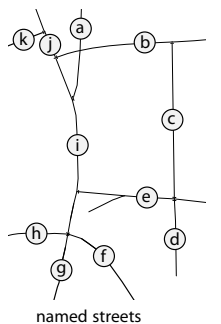

(b)

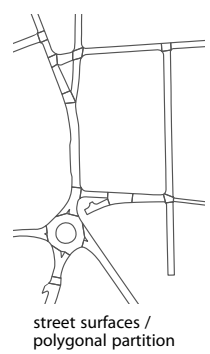

(f)

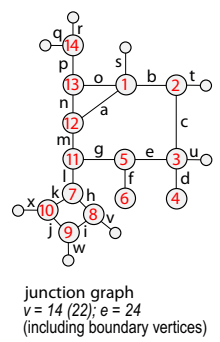

(j)

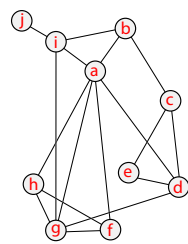

axial line graph

(n)

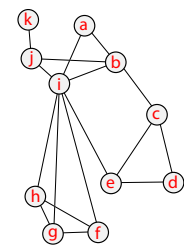

named street graph $v=10 ; e=16$ (c)

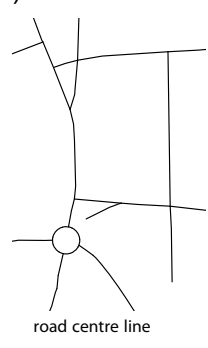

(g)

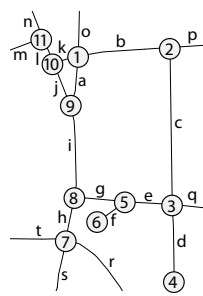

labelled generalised nodes and links

(k)

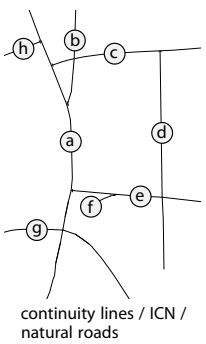

(o)

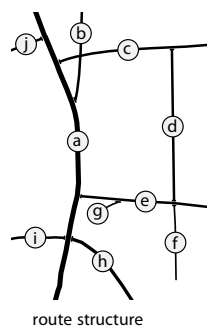

(d)

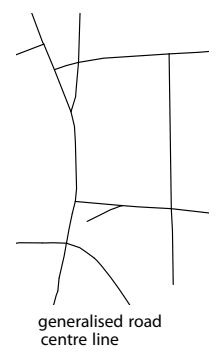

(h)

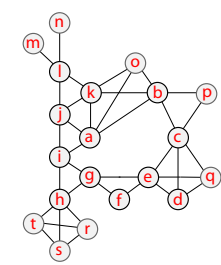

street-segment graph $v=12(20) ; e=19(35)$
(including boundary segments)

(I)

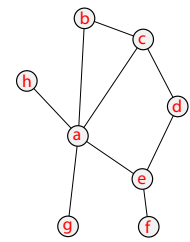

continuity line / ICN / natural road graph

(p)<smiles>OC1(O)OOC1(O)O</smiles>

route graph $v=10 ; e=11$

Fig. 1 From street environment data sets (top row), to street network models and their graph representations (second, third and fourth rows). The top row a-d shows different data sets used as input for creating street network models. Below are examples of different interpretations of streets as network models (first and third columns), with the junctions labelled with numbers and the street segments labelled with letters. Next to each of these (second and fourth columns) are the corresponding graph representations used for analysis, with the graph vertices labelled in red. Base mapping (C) Crown Copyright and Database Right (2018). Ordnance Survey (Digimap Licence) 
to as the 'node-link' representation by Steadman (2004), conflating street network model and graph representation in one concept, since the conversion from one to the other is so direct and intuitive. However, since any graph could be interpreted as a 'node-link' representation, we propose to call this (Fig. 1, f) the junction graph, in effect adopting the convention that the type of graph is named after the entity primarily represented by the vertices. This convention matches some existing usage and is applied throughout Fig. 1 and the rest of this paper.

As is clear from Fig. 1, a graph representation need not equate the linear paths of movement with graph edges. A street network analysis might take the streets themselves as the primary elements of study, and represent those as vertices in a graph, while the connections between streets (i.e. junctions or intersections) become edges in the corresponding graph. This representation (Batty 2004a, b), can be seen as equally logical and intuitive from its own point of view (Marshall 2016). In transportation, a graph where the street segment is the vertex and the junctions are edges has been used to include turn costs and restrictions in the model (Caldwell 1961; Winter 2002). This can be referred to as the street-segment graph (Fig. 1, h).

Similarly, a street network could be represented by an axial map (comprising axial lines, Fig. 1, i) (Hillier and Hanson 1984), and analyses carried out in which those axial lines are represented by the vertices in the graph, while intersections between axial lines are represented by edges (Fig. 1, j). Each other kind of network model discussed here has a corresponding graph (Fig. 1, k-p).

When using a node and link street network model, one can extract weights from the geometry of the network and assign them to the edges of the graph. In the case of the conventional junction graph, this weight is typically the length of the links. In the case of the street segments graph, this weight can be the angular deviation of the streets incident in a junction, or the sum of half the length of each link (Winter 2002). Using these weighted graphs of street segments as vertices it is possible to generate a range of alternative non-planar graph representations (Gil 2014; Figueiredo 2015a; Stavroulaki et al. 2017; Tomko et al. 2008) that correspond to continuous street network models.

\subsection{A Note on Terminology}

The dominant representation of street network models as graphs (links as edges, nodes as vertices) has been widely referred to as the primal representation (Porta et al. 2006b), while the alternative (aggregated streets as vertices, intersections as edges) has been referred to as the dual graph of the street network model (Porta et al. 2006a). This terminology is not only inconsistently used in the literature because the choice of which graph is primal depends on the starting point (see for example Batty 2004a p. 5), but is also unfortunate in particular due to assuming a narrow relationship between the street modelling process and the graph representation.

A mathematical dual graph has a precise, symmetrical involution relationship implying that if $\operatorname{dual}(A) \rightarrow B$, then $\operatorname{dual}(B) \rightarrow A-$ as is the case for planar graph duals, where the dimensionality of nodes and edges are reversed (e.g., Voronoi tessellation vs. Delaunay triangulation, Fig. 2, a). Because the relationship is symmetrical the graphs are duals of each other, and neither is strictly 'primal'. 


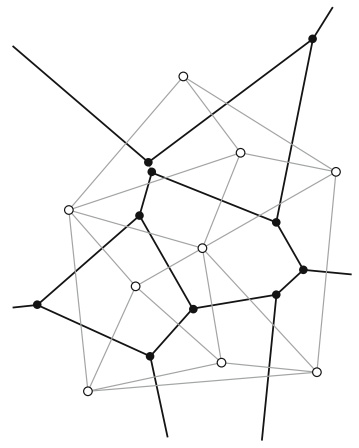

a) Voronoi - Delaunay duality

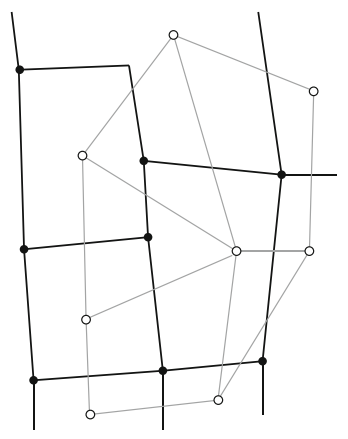

b) Street Junction - Block duality

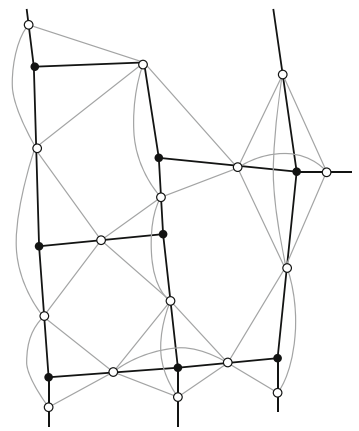

c) Street Junction - Segment duality

Fig. 2 Different examples of graph dual relationships

This duality may be perceived between the planar junctions graphs of the node and link street network model (junctions as vertices, streets as links) and the urban block adjacency graph defined by the streets segments as boundaries of polygonal areas (Fig. 2, b). The duality between the junctions graph and street segments graph (Fig. 2, c) is not mathematically the same, hence the term pseudo-dual employed by Winter (2002).

This dual relationship does, however, not hold with the so-called dual models of street networks noted above (Porta et al. 2006a), where the involution process is not symmetrical and leads to loss of information. The different approaches to modelling street networks based on the continuity of street segments (see Section 3) result in mostly non-planar graphs (Fig. 1, rows 3 to 4), and therefore there is no dual relationship as with the planar graphs in Fig. 2. Alternative terminology could be suggested (Marshall 2016) but a definitive conclusion on this issue awaits a dedicated review of all relevant kinds of graph and their relations.

\section{Creating and Analysing Street Network Models}

\subsection{Creating Street Network Models}

The creation of a street network model requires the choice of a data set to support its representation, but the reverse can also occur: the availability of a street network data set suggests the choice of a specific street network model. The widely available road centre line (RCL) data sets offer a specific representation of street segments between intersections (Fig. 1 top row, a-d), thus are directly usable for node-link models, and for junctions or street segment graphs (Fig. 1 row 2, e-h). Other levels of representation can be derived from such data, e.g. long curvilinear street segments can be broken into individual line segments (Stavroulaki et al. 2017) or axial lines (Fig. 1 i), or contiguous street segments can be aggregated into alternative continuous street representations (Fig. $1 \mathrm{k}, \mathrm{m}, \mathrm{o}$ ). However, while RCL are common, they should not be used without rigorous attention and preparation. They often contain 
errors, where the principle of interrupting street segments at intersections is not kept. They can also include numerous road infrastructure artefacts such as traffic islands, roundabouts, multiple lanes and sliproads at interchanges, that may not be meaningful in a given street network model. Different data sets available for the same location may be available - often from the same provider - offering different levels of generalisation (Fig. $1 \mathrm{~d}$ ) of the data based on e.g., cartographic considerations. These will lead to distinct analytical conclusions. While generalised data sets simplify the street network geometry and eliminate undesirable excess detail, they also limit what is included in the network, usually excluding pedestrian paths, public spaces, and minor roads that are of particular interest to street network studies.

In some street network approaches the street network is drawn manually to achieve specific representations of continuity (e.g., the axial map, Fig. 1, i). This approach is often considered subjective and laborious, limiting the reproducibility and spatial extent of the research. Nevertheless the researcher can choose the level of representation and what to include in the model as required by the purpose of the research.

\subsection{Analysing Street Network Models}

Ultimately, whatever the street network model adopted, a graph is created consisting of vertices and edges, eventually with some kind of weights (Table 1). This graph can then be analysed in different ways depending on the purpose of the study. Different analytic methods have been used, stemming from different disciplines - transport network analysis, network centrality, geographic accessibility, and typological classification. Some examples of what these methods represent and how they are applied to different street network graphs are now suggested for illustration.

Traversal Transport network analysis includes traversal problems, such as single journey routing (e.g., Caldwell 1961), service area calculation (e.g., Okabe and Kitamura 1996; Peponis et al. 2008), origin destination matrix calculation, and other route optimisation algorithms such as the travelling salesman problem. These analyses use the network as a means to get from A to B without measuring properties of the network elements themselves. They are therefore very much aligned with the transport network representation, and work best with disaggregate representations of street networks because they rely on individual routes and paths meandering through the network (Cardillo et al. 2006).

Global importance of network elements Network centrality, on the other hand, consists of a collection of algorithms from network science, such as degree, closeness, betweenness, or eigenvector centrality, that give a measure to every vertex in the graph (Newman 2003; Crucitti et al. 2006). These are indicators of the relative importance of graph vertices, as the number of connections (degree) to other vertices, or being close (lower average distance) to all others, or between (frequently on shortest routes) all others. These metrics are an intrinsic property of the network as object of study, and therefore very common in street networks and urban studies. Most network centrality measures can be calculated on any type of graph, irrespective of the 


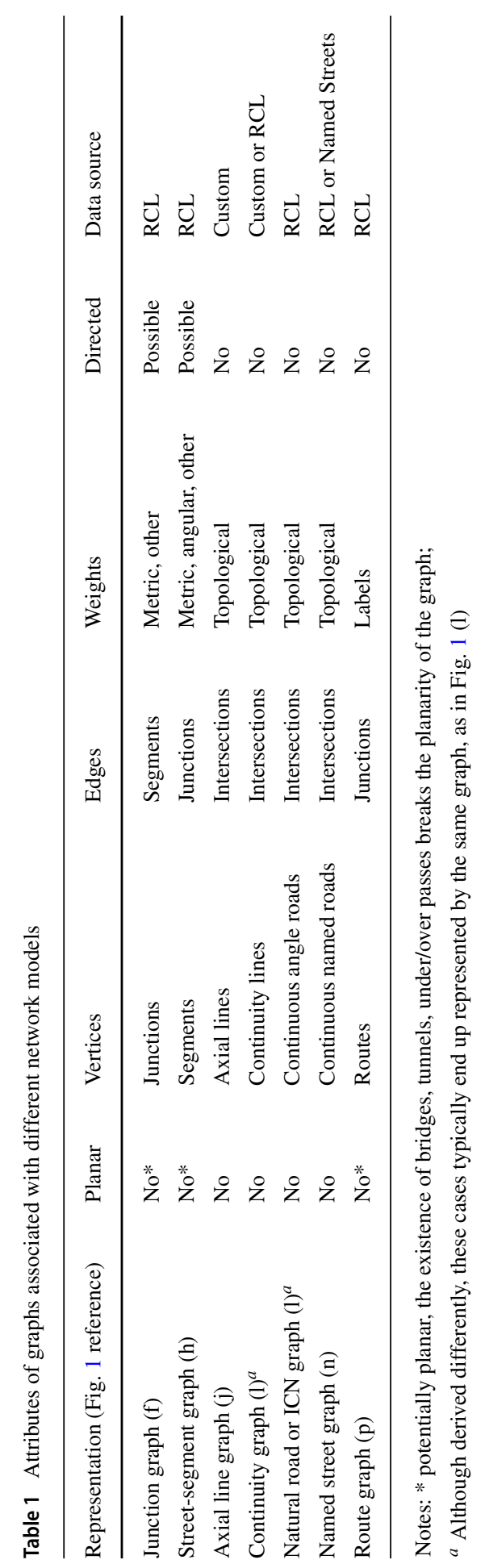


street network representation, as long as it meets required mathematical criteria, such as having a single connected component or a minimum meaningful size. The results and their interpretation are obviously sensitive to the modelling approach adopted (Gil 2017).

Network subgraphs A number of network measures can be calculated as the value of a location on the network in relation to opportunities within a cut-off distance or threshold, based on metric (e.g., Porta et al. 2006b) or topological (e.g., Hillier and Hanson 1984) distances, time, budget, quantity, size or perceived utility. They are similar to global measures except that the cut-off or threshold rule create a subgraph for each node in the system. In some cases they may include other features of the urban environment that are not part of the street network, e.g. land use (Ståhle et al. 2005; Sevtsuk and Mekonnen 2012). As with simpler transport network analysis, this type of indicator is most suited for disaggregate representations of street networks, and requires a diversity of weights and additional data sets (Miller 1999; Okabe and Kitamura 1996). However, it can also be performed in aggregated representations, such as the axial map, alone or combined with plots (Ståhle et al. 2005).

Typological analysis Finally, other network studies use the resulting model to identify typological characteristics of the different elements, streets or junctions. For this purpose a range of techniques are used, such as based on the route structure or geometric characteristics of the network elements (sinuosity of the streets, angles between links in junctions) for use in morphological analyses, street layout design or conservation planning, e.g. Marshall (2005), Kropf (2017), and Thomson and Richardson (1999).

It should be stressed that in principle every type of analysis could be made using every graph, but some are more meaningful than others. The more disaggregated representations, consisting of individual street elements such as junctions and segments, are perceived as providing a greater level of detail in the analysis, while the more aggregated models, combining street elements based on different notions of continuity, provide different types of structural properties and are mostly suitable for topological analysis of global patterns of centrality.

\section{Discussion and Outlook}

In this paper we have sketched out a number of issues that we believe are important to the ongoing practice and development of street network studies, in terms of representation, modelling and analysis. Over and above drawing attention to the distinctive characteristics of street networks (as opposed to other transport or spatial networks), a key contribution here was to discuss different treatments of street systems as networks, models and representations, including different levels of abstraction, and representation as different kinds of graphs. We have tried to articulate these different treatments in sufficient detail - commensurate with a concise overview - to alert the reader to the potential significance and value of considering them explicitly in street network studies. 
Treating a street system as a network is of itself a commitment to a paradigm, a modelling decision that influences how that street system is analysed, valued and ultimately designed or managed. There is a range of models of street network structures (Fig. 1), providing alternatives to the conventional model with junctions as nodes and street segments as links. In turn, these models may be differently represented as graphs (Table 1), which lend themselves to analyses for traversal, connectivity, and centrality. These analyses in turn reveal a range of local or global properties of individual streets or junctions, their aggregations including routes, or help understand the statistical properties of the whole street network.

While different traditions have established preferences when studying networks, we consider it valuable that different disciplines can learn from each other, and that all those traditions can consider the approaches noted in this article. We hope this will allow for a more informed, purposeful selectivity in street system modelling carved from this plurality of choices. Some models benefit from the simple and direct derivation from common data sources (RCL) using mainstream software tools and consequently are dominant in the scholarship tradition of network science. Yet, street networks in particular require a wider and more nuanced range of analytical approaches to capture the complex aspects of street environments and not purely their statistical properties as derived from just one particular model or representation.

As a way forward, we argue for a more unified and comprehensive attitude to street network studies. First, we argue for a greater awareness of the different approaches available. Even based on existing knowledge, it seems incumbent on researchers and analysts to be mindful of the different kinds of model and representation already available, and that we ought to reflect, specify, and justify our choices in terms of model and analysis more transparently.

In particular, we suggest that in street network studies the network modelling step is critical, and should be transparent, deliberate, and grounded in a theory. As a result, each study should be driven by a careful and documented modelling method. Such a model is an abstraction of the physical street network into the components and properties deemed relevant for the purpose of the study by the analyst. This involves its explicit translation into a graph representation, including definition of vertices, edges, weights, and the model's boundary, that are deemed adequate for the selected analysis, always bearing in mind that decisions leading to different graphs are inextricable with decisions about the weights or measures of those graphs. This process would ideally be open to, or at least informed by, the traditions across the urban disciplines. And, if required, it should lead to the adoption of different street network models for different aspects of the study, as they represent and provide insight into different aspects of street networks. The different models must be seen as complementary and not mutually exclusive.

Second, we encourage theoretical consolidation of the field, with more detailed comparative theoretical analyses of these models and analytical approaches, including how they relate to each other and to other traditions in science that study networks. This paper can be considered a start, but broader and deeper studies are suggested, that could clarify the relationships between the different kinds of street network models and different kinds of graph representations (Fig. 1; Table 1) and the analyses for which they are suited. This theoretical agenda could also be extended to 
relationships with other components of the urban system - landmarks, barriers, and their relationships in heterogeneous urban networks. This would be greatly assisted by a greater consistency in terminology.

Thirdly, we need greater understanding of the relative strengths and limitations of each approach in terms of applications to particular contexts, their measures and analyses, and their stringent validation and evaluation through empirical studies. This is critical in order to understand how the different approaches perform the same problem in different settings, and whether this multitude is enriching the field of urban research or rather creating confusion. A strengthened street network science can then help to inform applications to street network studies, including not only analysis but supporting network design, planning and management.

Open Access This article is distributed under the terms of the Creative Commons Attribution 4.0 International License (http://creativecommons.org/licenses/by/4.0/), which permits unrestricted use, distribution, and reproduction in any medium, provided you give appropriate credit to the original author(s) and the source, provide a link to the Creative Commons license, and indicate if changes were made.

\section{References}

Anderson S (1978) On streets. The MIT Press, Cambridge Massachusetts

Anez J, Barra TDL, Perez B (1996) Dual graph representation of transport networks. Transp Res Part B: Methodol 30(3):209 - 216. https://doi.org/10.1016/0191-2615(95)00024-0, http://www.sciencedirect. com/science/article/pii/0191261595000240

Barthelemy M (2011) Spatial networks. Phys Rep 499(1-3):1-101. https://doi.org/10.1016/j.physrep.2010. 11.002 http://www.sciencedirect.com/science/article/pii/S037015731000308X

Batty M (2004a) A new theory of space syntax. CASA working papers series WP75

Batty M, Rana S (2004b) The automatic definition and generation of axial lines and axial maps. Environ Plann B: Plann Des 31(4):615-640

Brandes U, Robins G, McCranie A, Wasserman S (2013) What is network science? Netw Sci 1(1):1-15

Butts CT (2009) Revisiting the foundations of network analysis. Science 325(5939):414-416

Caldwell T (1961) On finding minimum routes in a network with turn penalties. Commun ACM 4(2):107108

Cardillo A, Scellato S, Latora V, Porta S (2006) Structural properties of planar graphs of urban street patterns. Phys Rev E 73(6):066,107. https://doi.org/10.1103/PhysRevE.73.066107,00269

Courtat T, Gloaguen C, Douady S (2011) Mathematics and morphogenesis of cities: a geometrical approach. Phys Rev E 83(3):036,106

Crucitti P, Latora V, Porta S (2006) Centrality measures in spatial networks of urban streets. Phys Rev E 73(3):036,125:1-036,125:5. https://doi.org/10.1103/PhysRevE.73.036125,00414

Ducruet C, Beauguitte L (2014) Spatial science and network science: Review and outcomes of a complex relationship. Netw Spat Econ 14(3-4):297-316

Figueiredo L (2015a) A unified graph model for line and segment maps. In: Proceedings of the 10th International Space Syntax Symposium. University College London, London, pp 146:1-146:11, http://www.sss10.bartlett.ucl.ac.uk/wp-content/uploads/2015/07/SSS10_Proceedings_146.pdf

Figueiredo L, Amorim L (2005b) Continuity lines in the axial system. In: Proceedings of the 5th International Space Syntax Symposium. TU Delft, Delft http://www.spacesyntax.tudelft.nl/media/ Long

Garrison WL, Marble DF (1962) The structure of transportation networks. Technical report, Northwestern University Evanston ILL

Gehl J (2011) Life between buildings: using public space. Island Press, Washington

Giacomin DJ, Levinson DM (2015) Road network circuity in metropolitan areas. Environ Plann B: Plann Des 42(6):1040-1053

Gil J (2014) Analyzing the Configuration of Multimodal Urban Networks. Geogr Anal 46:368-391. https://doi.org/10.1111/gean.12062, http://onlinelibrary.wiley.com/doi/10.1111/gean.12062/abstract 
Gil J (2017) Street network analysis "edge effects": Examining the sensitivity of centrality measures to boundary conditions. Environ Plann B: Urban Anal City Sci 44(5):819-836. https://doi.org/10.1177/ 0265813516650678

Haggett P, Chorley RJ (1969) Network analysis in geography. Edward Arnold, London

Hahmann S, Miksch J, Resch B, Lauer J, Zipf A (2018) Routing through open spaces-a performance comparison of algorithms. Geo-spatial Inf Sci 21(3):247-256

Hillier B, Hanson J (1984) The social logic of space. Cambridge University Press, Cambridge

Hillier B, Penn A, Hanson J, Grajewski T, Xu J (1993) Natural movement: or, configuration and attraction in urban pedestrian movement. Environ Plann B: Plann Des 20(1):29-66

Jiang B, Claramunt C (2004a) A structural approach to the model generalization of an urban street network. GeoInformatica 8(2):157-171

Jiang B, Claramunt C (2004b) Topological analysis of urban street networks. Environ Plann B: Plann Des 31(1):151-162

Jiang B, Zhao S, Yin J (2008) Self-organized natural roads for predicting traffic flow: a sensitivity study. J Stat Mech: Theory Exp 2008(07):P07,008

Jiang B, Okabe A (2014) Different ways of thinking about street networks and spatial analysis. Geogr Anal 46(4):341-344

Kansky KJ (1963) Structure of transportation networks: relationships between network geometry and regional characteristics, Chicago

Kivelä M, Arenas A, Barthelemy M, Gleeson JP, Moreno Y, Porter MA (2014) Multilayer networks. J Compl Netw 2(3):203-271

Kropf K (2008) Route structure analysis. Urban Des 105:10-11

Kropf K (2017) The handbook of urban morphology. Wiley, New York

Lagesse C, Bordin P, Douady S (2015) A spatial multi-scale object to analyze road networks. Netw Sci 3(1):156-181

Louf R, Barthelemy M (2014) A typology of street patterns. J Royal Soc Interface 11(101):20140,924

Mandloi D, Thill JC (2010) Object-oriented data modeling of an indoor/outdoor urban transportation network and route planning analysis. In: Geospatial Analysis and Modelling of Urban Structure and Dynamics. Springer, pp 197-220

Marshall S (2005) Streets and patterns. Routledge, London and New York

Marshall S (2016) Line structure representation for road network analysis. J Transp Land 9(1):29-64. https://doi.org/10.5198/jtlu.2015.744

Masucci AP, Stanilov K, Batty M (2014) Exploring the evolution of London's street network in the information space: a dual approach. Phys Rev E 89(1):012,805

Meeteren M, Neal Z, Derudder B (2016) Disentangling agglomeration and network externalities: a conceptual typology. Pap Reg Sci 95(1):61-80

Miller HJ (1999) Measuring Space-Time Accessibility Benefits within Transportation Networks: Basic Theory and Computational Procedures. Geogr Anal 31(2):187-212. https://doi.org/10.1111/j.1538-4632. 1999.tb00976.x

Newman ME (2003) The structure and function of complex networks. SIAM Rev 45(2):167-256

Okabe A, Kitamura M (1996) A Computational Method for Market Area Analysis on a Network. Geogr Anal 28(4):330-349. https://doi.org/10.1111/j.1538-4632.1996.tb00939.x

Peponis J, Bafna S, Zhang Z (2008) The connectivity of streets: reach and directional distance. Environ Plann B: Plann Des 35(5):881-901

Porta S, Crucitti P, Latora V (2006a) The network analysis of urban streets: A dual approach. Physica A 369(2):853-866. http://www.citebase.org/cgi-bin/citations?id=oai:arXiv.org:cond-mat/0411241

Porta S, Crucitti P, Latora V (2006b) The network analysis of urban streets: a primal approach. Environ Plann B: Plann Des 33(5):705-725

Sadik-Khan J (2017) Streetfight: handbook for an urban revolution. Penguin, Baltimore

Sevtsuk A, Mekonnen M (2012) Urban network analysis. Rev Int Géomatique-n 287:305

Ståhle A, Marcus L, Karlström A (2005) Place Syntax- Geographic Accessibility with Axial Lines in GIS. In: Proceedings of the 5th International Space Syntax Symposium. TU Delft, Delft, pp 131144

Stavroulaki G, Marcus L, Berghauser Pont M, Nilsson L (2017) Representations of Street Networks in Space Syntax: Towards flexible maps and multiple graphs. In: 11th International Space Syntax Symposium, Lisbon. http://www.11ssslisbon.pt/docs/proceedings/papers/174.pdf

Steadman P (2004) Developments in space syntax. Environ Plann B: Plann Des 31(4):483-486 
Strano E, Nicosia V, Latora V, Porta S, Barthélemy M (2012) Elementary processes governing the evolution of road networks. Sci Rep 2:296

Thomson RC, Richardson DE (1999) The 'Good Continuation' Principle of Perceptual Organization applied to the Generalization of Road Networks. In: Proceedings of the ICA 19th International Cartographic Conference, pp 1215-1223, 00196

Tomko M, Winter S, Claramunt C (2008) Experiential hierarchies of streets. Comput Environ Urban Syst 32(1):41-52

Tomko M, Winter S (2013) Describing the functional spatial structure of urban environments. Comput Environ Urban Syst 41:177-187. https://doi.org/10.1016/j.compenvurbsys.2013.05.002

Turner A (2007) From axial to road-centre lines: a new representation for space syntax and a new model of route choice for transport network analysis. Environ Plann B: Plann Des 34(3):539-555

Winter S (2002) Modeling costs of turns in route planning. GeoInformatica 6(4):345-361

Xie F, Levinson D (2007) Measuring the structure of road networks. Geogr Anal 39(3):336-356

Yerra BM, Levinson DM (2005) The emergence of hierarchy in transportation networks. Ann Reg Sci 39(3):541-553

\section{Affiliations}

\section{Stephen Marshall ${ }^{1}$ D . Jorge Gil $^{2} \cdot$ Karl Kropf $^{3} \cdot$ Martin Tomko $^{4} \cdot$ Lucas Figueiredo $^{5}$}

Jorge Gil

jorge.gil@chalmers.se

Karl Kropf

kkropf@brookes.ac.uk

Martin Tomko

tomkom@unimelb.edu.au

Lucas Figueiredo

lucasfigueiredo@ct.ufpb.br

1 Bartlett School of Planning, University College London (UCL), London, UK

2 Department of Architecture and Civil Engineering, Chalmers University of Technology, Göteborg, Sweden

3 Oxford Brookes University, Oxford, UK

4 The University of Melbourne, Parkville, Australia

5 Universidade Federal da Paraíba (UFPB), João Pessoa - PB, Brazil 